\title{
OPTICAL LINE EMISSION FROM IO
}

\author{
ROBERT A. BROWN
}

Center for Earth and Planetary Physics, Harvard University, Cambridge, Mass., U.S.A.

\begin{abstract}
In early summer of 1972, we discovered an anomalous brightness in the spectrum of Io near the sodium D-lines. This effect is revealed to be time-varying emission by free sodium atoms on Io.
\end{abstract}

A year and a half ago there was no reason to expect the new and the unusual in the visible spectra of the Galilean satellites of Jupiter. They were, after all, discovered in January, 1610, and they have been easy objects of spectroscopic studies for more than 50 years. Devoid as those moons are of any yet sensible atmosphere, one would expect to find reflected from them the detailed spectrum of the Sun, variously tempered by broad color differences between the satellites (Johnson and McCord, 1970; Johnson, 1971).

Io is the innermost Galilean satellite. It is known to demonstrate two unusual behaviors: a possible post-eclipse brightening (Binder and Cruikshank, 1964) and a modulation of Jovian decametric radiation (Bigg, 1964). In the early summer of 1972 we discovered an anomalous brightness in the spectrum of Io near the sodium D-lines, which in the solar spectrum are marked by strong absorptions. A series of subsequent observations have revealed this effect to be time-varying emission by free sodium atoms on Io. This is a brief review of our current knowledge of this phenomenon.

Figure 1 shows interferometric spectra of two satellites, Io and Ganymede. The bold

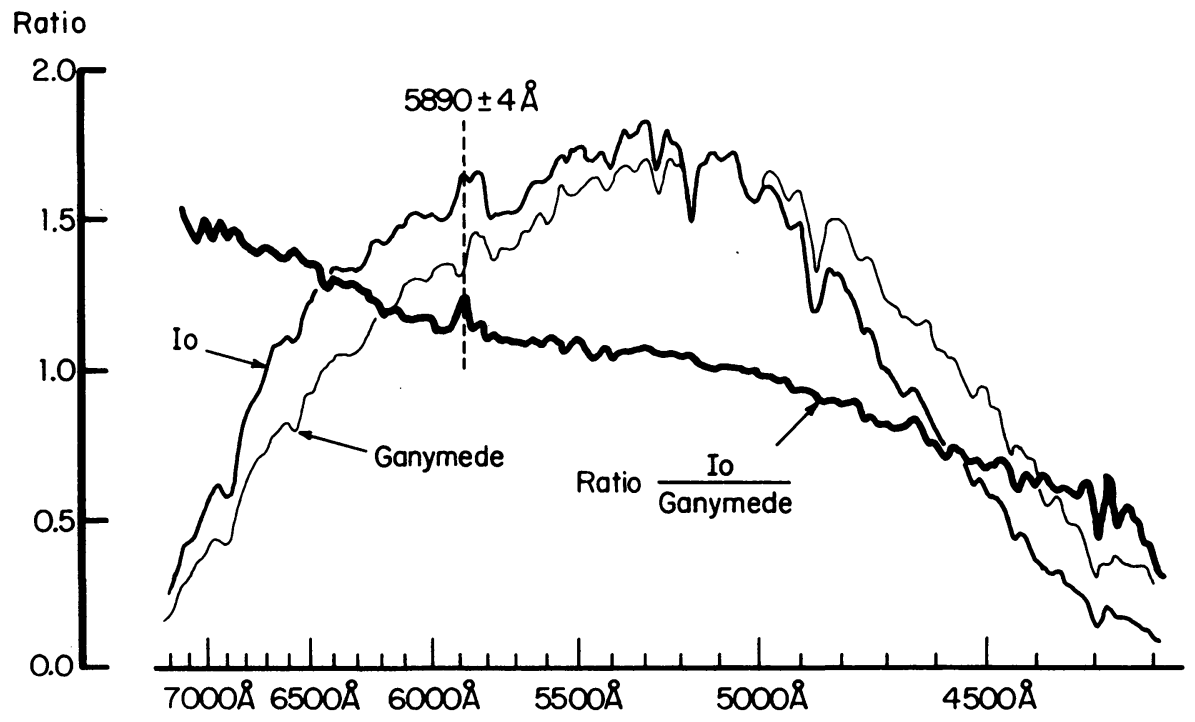

Fig. 1. The emission feature shown in the spectrum of Io when it is normalized to the spectrum of Ganymede. Data taken with interferometer. 
line shows the (arbitrarily normalized) ratio between those two spectra and shows the sharp brightening of Io at the D-lines, which are not separated at this resolution. Note that other deep Fraunhofer features in the original spectra are neatly cancelled in the ratio.

Ratio spectra in each combination for all four of the Galilean satellites are shown in Figure 2. These spectra were taken on a different night and were truncated by a broad optical filter which preceded the spectrometer. Note that the anomaly is present only in the spectrum of Io. A level ratio would indicate the normal solar D strength of $-1.2 \AA$ equivalent width in absorption. In the two similar examples of Figures 1 and 2 Io had a net D-line strength of about $2.5 \AA$ in emission.

Figure 3 shows a closer look with a high resolution spectrograph on a different night. A sharp and slightly shifted emission line is seen in each of the broad solar D absorptions. A Doppler shift analysis on the emission features proceeded as follows. A wavelength calibration was obtained by fitting the positions of the 10 indicated telluric water vapor lines. Figure 4 shows the solar absorption lines interpolated and subtracted from the observed spectrum to isolate the emission lines. In Figure 5

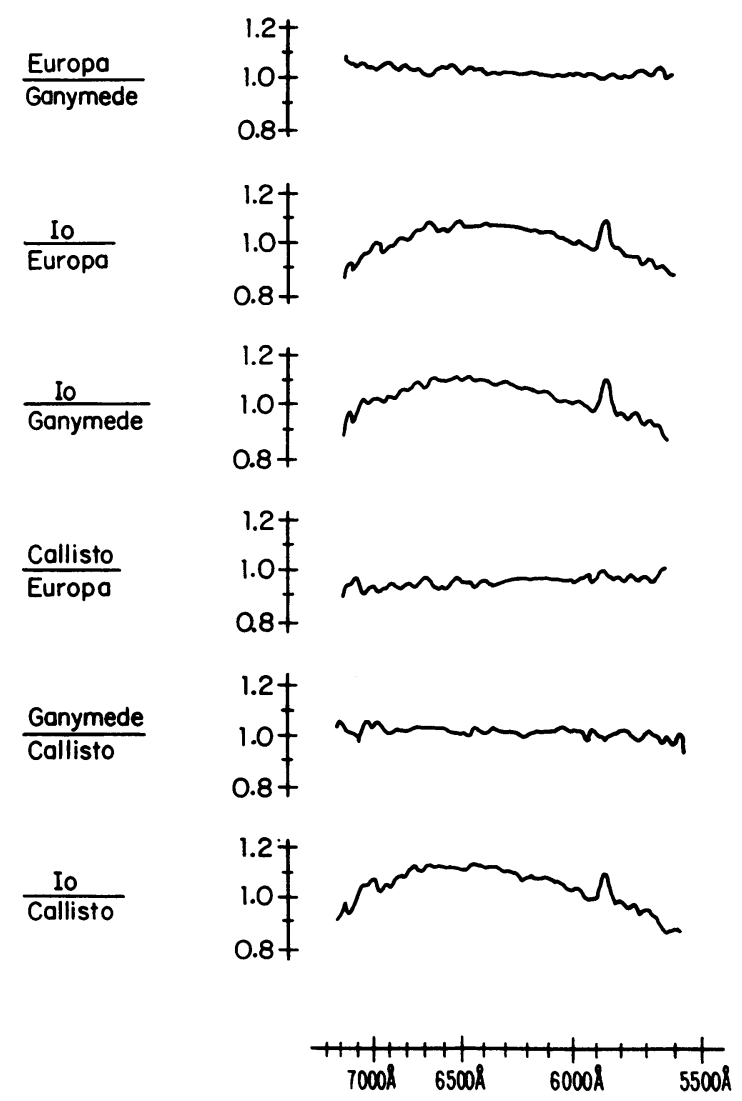

Fig. 2. Ratio spectra of Galilean satellites. May 25, 1972, 04:38-08:06 UT. 
D1

$5895.92 \AA$
02

$5889.95 \AA$

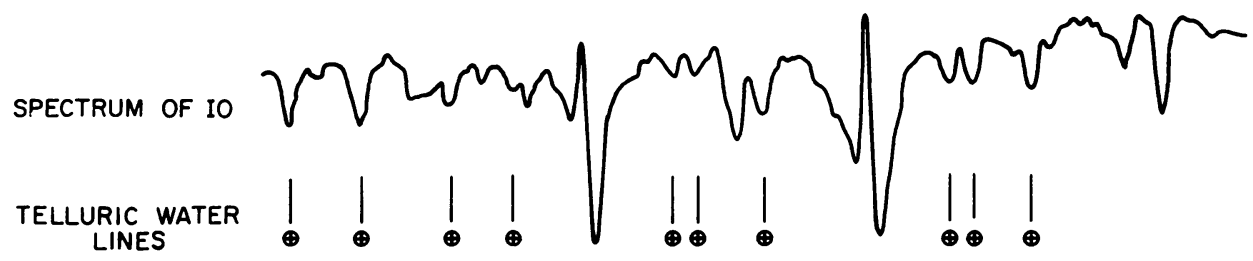

Fig. 3. High resolution spectrum of Io taken with echelle spectrograph. July 6, 1973, 10:19-10:49 UT.

RECOROED SPECTRUM

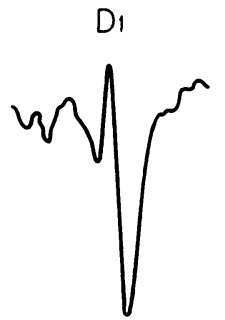

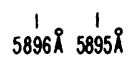

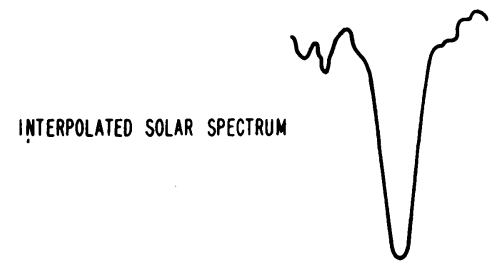

$5896 \stackrel{1}{1} 5895 \AA$

OIFFERENCE SPECTRUM

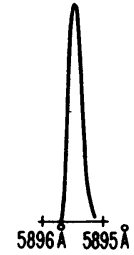

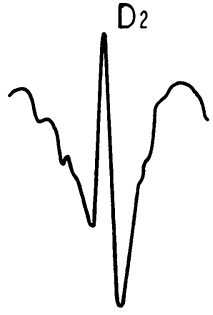

$5890 \AA 51180$

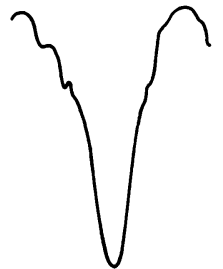

$5890 \AA \quad 5889 \AA$

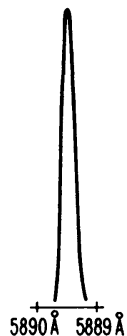

Fig. 4. Solar absorption lines interpolated and substracted from the observed spectrum to isolate the emission lines. 


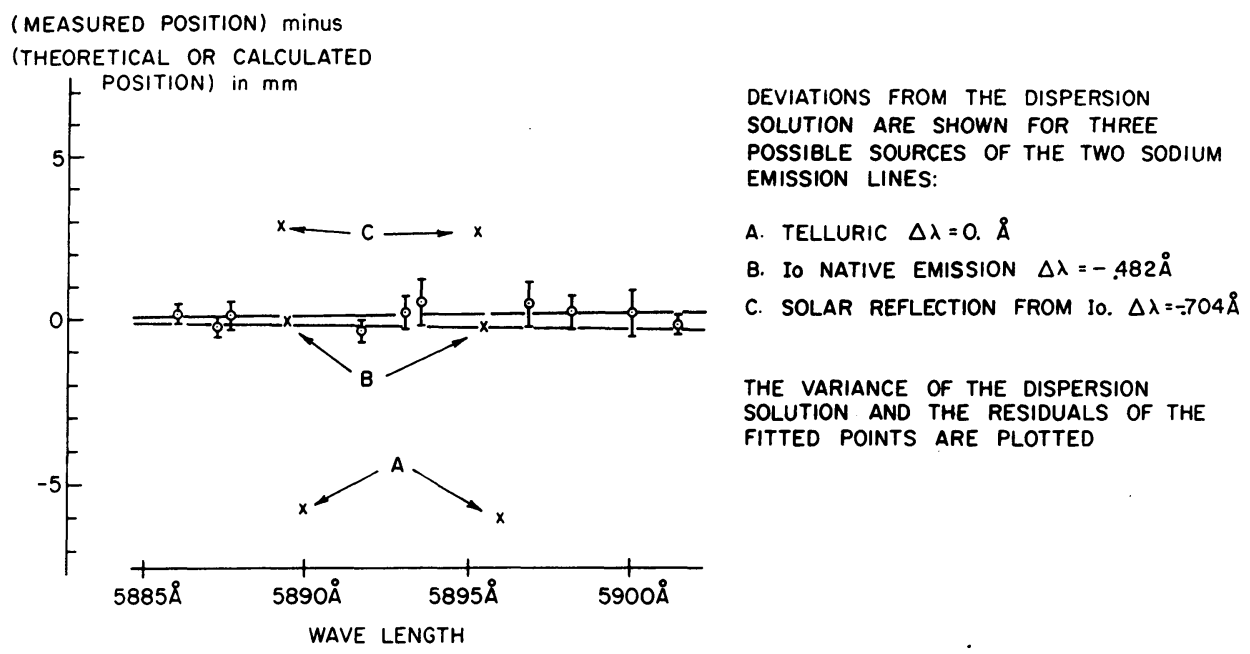

Fig. 5. Doppler shift identification of Io as the emission source.

the variance of the wavelength calibration is shown by the lines, and the residuals at the fitted points are plotted. The relative velocities of all the bodies in question are known for the observation period. The $x$ 's show the residuals when the emission lines are variously interpreted as originating on the Earth, the Sun or Io. Io is confirmed as the source of the emission.

The ratio of the equivalent widths of the two D-line components in the high resolution spectrum is $D_{2} / D_{1}=1.23 \pm 0.08$. A value of 2 is expected for a source in which fine structure sublevels populated according to their multiplicity and in which selfabsorption may be neglected. On the premise that there is indeed considerable selfabsorption, the observed ratio and line width can be used to calculate the sodium abundance, but such an estimate is model dependent. A simple view is that the absorption and emission line profiles are the same, and that the two processes are occurring in the same uniform layer, with resonant re-emission neglected. The derived optical thickness is $3.5_{-2}^{+3}$ in the center of the $D_{1}$ line, and the sodium column abundance is $\left(1.8_{-0.6}^{+1.8}\right) \times 10^{12} \mathrm{~cm}^{-2}$.

The strength of this emission from Io is varying in time. Figure 6 shows 17 independent measurements with 4 different spectrometers of the net D-line strength vs the departure of Io from superior geocentric conjunction. These measurements show a roughly uniform distribution when they are plotted against sub-Io Jovian longitude.

We are initiating two observational programs to study this interesting effect on Io. High resolution spectroscopy will be used to examine other sodium lines and to search for line emission by other species. Secondly, we will monitor the strength of the D-line emission and seek correlations with other phenomena. In all probability this discovery will prove a useful tool in probing both the Jovian environment and the conditions on this fascinating satellite. 


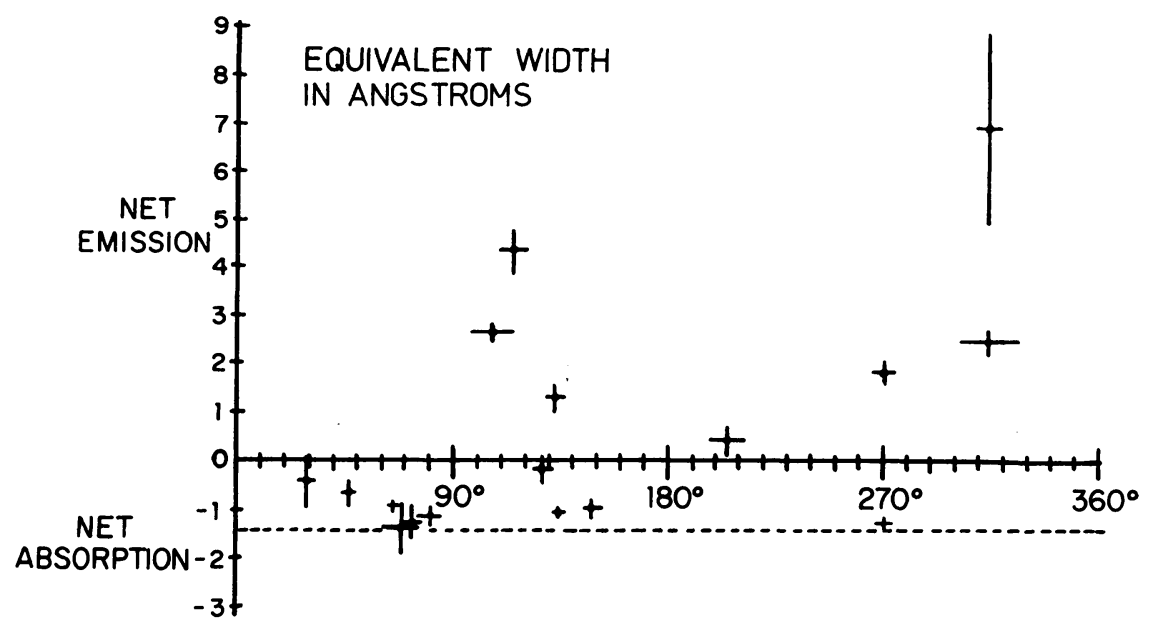

Fig. 6. Net strength of sodium D-lines vs departure from superior geocentric conjunction. Measures ments with four spectrometers with different dispersions and spacial apertures are shown.

\section{Acknowledgements}

Richard Goody suggested our first work on the Galilean satellites, which resulted in this discovery. Lawrence Mertz constructed the interferometer which was used, and he has been tireless in encouraging and assisting this work. Roger Schatel, Frank Murcray and David Latham have assisted with observations and data reduction. Frederic Chaffee is collaborating in the high resolution spectroscopic observations. A spectrum kindly taken by Helmut Abt is included in Figure 6.

This research was funded by the Atmospheric Sciences Section of the National Science Foundation under Grant No. GA-33990X.

\section{References}

Bigg, E. K.: 1964, Nature 203, 1008.

Binder, A. B. and Cruikshank, D. P.: 1964, Icarus 3, 299.

Johnson, T. V.: 1971, Icarus 14, 94.

Johnson, T. V. and McCord, T. B.: 1970, Icarus 13, 37. 